\title{
Correlations probed in direct two-nucleon removal reactions
}

\author{
E. C. Simpson and J. A. Tostevin \\ Department of Physics, Faculty of Engineering and Physical Sciences, University of Surrey, \\ Guildford, Surrey GU2 7XH, United Kingdom
}

(Received 25 July 2010; published 29 October 2010)

\begin{abstract}
Final-state-exclusive momentum distributions of fast, forward-traveling residual nuclei, following two-nucleon removal from fast secondary radioactive beams of projectile nuclei, can and have now been measured. Assuming that the most important reaction mechanism is the sudden direct removal of a pair of nucleons from a set of relatively simple, active shell-model orbital configurations, such distributions were predicted to depend strongly on the total angular momentum $I$ carried by the two nucleons - the final-state spin for spin $0^{+}$projectiles. The sensitivity of these now-accessible observables to specific details of the (correlated) two-nucleon wave functions is of importance. We clarify that it is the total orbital angular momentum $L$ of the two nucleons that is the primary factor in determining the shapes and widths of the calculated momentum distributions. It follows that, with accurate measurements, this dependence upon the $L$ makeup of the two-nucleon wave functions could be used to assess the accuracy of (shell- or many-body-) model predictions of these two-nucleon configurations. By using several tailored examples, with specific combinations of active two-nucleon orbitals, we demonstrate that more-subtle structure aspects may be observed, allowing such reactions to probe and/or confirm the details of theoretical model wave functions.
\end{abstract}

DOI: 10.1103/PhysRevC.82.044616

PACS number(s): 24.50.+g, 23.20.Lv, 21.60.Cs

\section{INTRODUCTION}

The momentum distributions of the residual nuclei, following the removal of a single nucleon from a fast radioactive secondary beam, offer sensitive probes of both strongly bound and weakly bound single-particle structure near the (asymmetric) Fermi surfaces of neutron-rich and neutron-deficient nuclei. Specifically, the shapes and widths of the exclusive residue momentum distributions were shown to be characteristic of the orbital angular momentum of the removed nucleon [1-5].

The simplest generalization to the case of direct two-nucleon removal is to describe the wave function of the two nucleons in the projectile by a product of nucleon wave functions in assumed single-particle orbitals. Doing so, the two nucleons are uncorrelated, other than both being bound to the same core [6,7]. The heavy-residue longitudinal momentum distributions in this limit, being essentially the convolution of those of the single nucleons, depend on the assumed quantum numbers of the two nucleons but, in the absence of explicit antisymmetrization or total angular momentum coupling of the two nucleons, are not characteristic of specific residue final states [7].

More recent theoretical developments now treat fully the shell-model correlations of the two removed nucleons in the projectile many-body wave function [8,9]. In the fully correlated models the product of nucleonic wave functions is replaced by the shell-model two-nucleon overlap, incorporating (i) the two-nucleon parentage coefficients with respect to each residue final state (the two-nucleon amplitudes, or TNAs), (ii) proper antisymmetrization of the two removed nucleons, and (iii) proper angular momentum coupling.

The resulting theoretical description, and the insights developed here, are equally valid for reactions that remove two loosely or strongly bound nucleons. However, as has been discussed elsewhere [8], in the case of removal of two loosely bound nucleons the direct removal cross sections, of interest here, will be overwhelmed (experimentally) by indirect reaction (one-nucleon removal plus evaporation) events. See, for example, Ref. [10] for a quantitative consideration of the direct and indirect two-neutron removal reaction contributions along the neutron-rich carbon isotopic chain. For these reasons we restrict our attention to examples for which the removed nucleons are strongly bound, where the indirect removal paths are effectively closed, and for which the direct cross sections are accessible experimentally.

Demonstrative test cases, for example, in Ref. [11], assumed the two nucleons originated from a single orbital-a pure configuration. In this limit the TNAs enter only as a multiplicative (spectroscopic-like) factor and thus the new and interesting characteristics of the residue momentum distribution are a result of the correlations arising from antisymmetrization and angular momentum coupling.

These developments demonstrate the potential of twonucleon removal for exotic nucleus spectroscopy, showing the final-state-exclusive residue nucleus momentum distributions to have shapes and widths that are characteristic of the total angular momentum, $I$, carried by the removed pair of nucleons - and permitting final-state spin assignments to be made [12]. For the spin $J_{i}=0^{+}$projectile nucleus examples used in Ref. [11], there was a high sensitivity of the residue momentum distributions to the final-state total angular momentum $J_{f}=I$. Moreover, the shapes of these calculated distributions were robust with respect to variations of other key structure and reaction parameters, such as the nucleon separation energy. Further, the consideration of pure configuration examples (e.g., two protons, assumed removed from a single active $\pi 1 d_{5 / 2}$, $\pi 1 d_{3 / 2}$, or $\pi 2 s_{1 / 2}$ orbital) showed considerable insensitivity of the two-nucleon removal distributions to these individual nucleon quantum numbers, in stark contrast to results from single-nucleon removal reactions where the orbital angular momentum is critical. 
Thus, although the two-nucleon removal process is powerful for final-state spin spectroscopy in very exotic systems, its sensitivity to and ability to probe finer details of the shell-model wave functions and the two-nucleon configurations and correlations therein remain less clear. Already in Fig. 5 of Ref. [7], for the case of two-proton removal from ${ }^{28} \mathrm{Mg}$, using the fully correlated shell-model wave functions for the transitions to the first two low-lying ${ }^{26} \mathrm{Ne}\left(2^{+}\right)$ final states, one observes momentum distributions with different widths, demonstrating a sensitivity beyond the finalstate spin. Our objective here is to elucidate this sensitivity of the calculated residue momentum distributions to the particular two-nucleon configurations present and to understand the sensitivity to the combination of orbitals involved for a given value of the pair's total angular momentum $I$.

In contrast to two-nucleon transfer reactions, such as the $\left(p,{ }^{3} \mathrm{H}\right)$ reaction, wherein the $\left\langle\left. p\right|^{3} \mathrm{H}\right\rangle$ light-ion structure vertex preferentially selects the pickup of a spin-singlet $(S=0)$ neutron pair, the two-nucleon removal mechanism is not explicitly selective in the nucleon spins [8]. Both spin-singlet and spin-triplet components of the two-nucleon overlap will be probed and, under the assumption that the residue and nucleontarget interactions (and $S$-matrices) are spin-independent, the $S=0$ and $S=1$ terms contribute incoherently to the reaction yield. We show specifically, in the same way that single-nucleon removal is sensitive principally to the orbital angular momentum $\ell$ rather than the total angular momentum $j$ of the nucleon, that the two-nucleon removal reaction momentum distributions are sensitive to the components in the two-nucleon overlap with a given value of total orbital angular momentum $L, L=\ell_{1}+\ell_{2}$. The presence and relative strengths of these $L$ components are determined via the shell-model overlaps and their TNAs. Because for a spin-zero projectile the residue has total spin $J_{f}$, with $\boldsymbol{J}_{f}=\boldsymbol{L}+\boldsymbol{S}$, the total spin content of the overlap is determined by the nuclear structure. However, all spin components present are sampled by the reaction mechanism.

Recognizing the sensitivity to $L$ allows greater probing of the shell-model wave function, particularly in states where the mixing of several available nucleon configurations may be weak. Specific examples can exhibit particularly strong sensitivity to the orbital combinations of the pair. In cases of strong mixing and sharing of strength among several orbitals a particular value of $L$ may nevertheless be favored. In such cases the residue momentum distribution will be characteristic of the details of the states populated and not simply of the total angular momentum value $I$ of the nucleon pair. Of particular interest are different regions of $A$ and $Z$ that affect the originating, active orbitals of the two nucleons.

In Sec. II we outline the approximations assumed and then present the required formalism for residue momentum distributions within the $L S$-coupling scheme. We retain isospin labels for clarification of the underlying symmetries. In Sec. III the importance of the total orbital angular momentum is elucidated by a detailed consideration of the spatial and angular correlations of the two nucleons that are inherent in the two-nucleon overlap function. This analysis also provides insight into the possible sensitivity to the mixing of orbitals across major shells. In Sec. IV we then consider particular examples with projectiles of different $A$ and $Z$, where interesting effects are predicted. Examples look at specific final states that could be populated in two-nucleon removal from the $p$ shell, ${ }^{12} \mathrm{C}(-n p)$, the $s d$-shell, ${ }^{26} \mathrm{Si}(-2 n)$, and also the $s d p f$-cross-shell situation in ${ }^{54} \mathrm{Ti}(-2 p)$. We summarize the article and draw conclusions in the final section.

\section{FORMALISM}

We discuss the sudden, direct removal of two nucleons from a fast projectile beam incident on a light nuclear target at energies of order $80 \mathrm{MeV}$ per nucleon and greater. In this intermediate energy range there have been extensive (positive) assessments of the validity and accuracy of the suddenadiabatic and eikonal reaction dynamical approximations (see, for example, Sec. 3.5 of Ref. [13] and references therein) and of the theoretical ingredients used in their implementation, such as the importance of Pauli blocking on the effective nucleon-nucleon interaction used [14]. These wide-ranging assessments, carried out within the one-nucleon stripping and breakup contexts, remain valid in the present analysis, for example, the role of strong absorption between the projectile and the target in reducing the effective reaction time and the energy for validity of the sudden (fast adiabatic) approximation [15].

We first briefly discuss the salient features of the approach, previously developed in detail in Refs. [8,9,11]. Our emphasis here is on the use of the $L S$-coupling representation to describe the two-nucleon structure overlaps and to derive the expressions for the residue momentum distributions in this basis. We consider only those (stripping) reaction mechanism events in which the two removed nucleons interact inelastically with the target nucleus. The role on the cross sections and momentum distributions of the other major class of events (diffraction-stripping), where one of the nucleons is removed by an elastic interaction with the target, were discussed fully in Refs. [9] and [11], respectively. As was shown there, events from this second mechanism give residue momenta that are essentially identical to those of the stripping mechanism. These conclusions remain unchanged and are not repeated here.

We assume the projectile nucleus to be an antisymmetrized $A+2$-body (shell-model) system, denoted by $\Psi_{i} \equiv \Psi_{J_{i} M_{i} T_{i} \tau_{i}}(A, 1,2)$, carrying total angular momentum $J_{i}$ and isospin $T_{i}$. In a high-speed collision with the light target, two nucleons may be removed to produce an $A$-body reaction residue in a final state $f$, often referred to as the core state for simplicity. This final core state is $\Phi^{(F)} \equiv \Phi_{J_{f} M_{f} T_{f} \tau_{f}}(A)$. Each residue final state is denoted by $f$, while $F \equiv\left(f, M_{f}\right)$ is used to refer to a state with a specific angular momentum projection $M_{f}$. One is reminded that the final states of the two nucleons and of the target nucleus are unobserved and that the observables discussed are inclusive with respect to these degrees of freedom.

\section{A. Two-nucleon overlap}

The direct reaction probes the two-nucleon overlap

$$
\begin{aligned}
\Psi_{i}^{(F)} & \equiv \Psi_{J_{i} M_{i} T_{i} \tau_{i}}^{(F)}(1,2) \\
& \equiv\left\langle\Phi^{(F)}(A) \mid \Psi_{i}(A, 1,2)\right\rangle
\end{aligned}
$$




$$
\begin{aligned}
= & \sum_{I \mu T \alpha} C_{\alpha}^{I T}\left(I \mu J_{f} M_{f} \mid J_{i} M_{i}\right) \\
& \times\left(T \tau T_{f} \tau_{f} \mid T_{i} \tau_{i}\right)\left[\overline{\psi_{\beta_{1}}(1) \otimes \psi_{\beta_{2}}(2)}\right]_{I \mu}^{T \tau},
\end{aligned}
$$

where $\Psi_{i}$ and $\Phi^{(F)}$ were defined previously. The signed TNAs $C_{\alpha}^{I T} \equiv C_{\alpha}^{J_{i} J_{f} I T_{i} T_{f} T}$ are taken from shell-model calculations. They express the parentage (amplitudes) for finding each twonucleon configuration $\alpha$ and residue final state $f$ in the overlap with the projectile initial state $i$, assumed to be the ground state. The two-nucleon configurations index, $\alpha \equiv\left[\beta_{1}, \beta_{2}\right]$, denotes the spherical quantum numbers of the single-particle states occupied by the nucleon pair. Hence, $\beta \equiv n \ell j$. Note that the amplitudes $C_{\alpha}^{I T}$ refer to a specific $i \rightarrow f$, initial to final-state transition, which is understood implicitly.

The details of the shell-model calculations (e.g., the model spaces and interactions) used to construct these overlaps are presented with each relevant example in the later sections.

Expressed in $L S$ coupling, the antisymmetrized twonucleon wave function in Eq. (1) is

$$
\begin{aligned}
& {\left[\overline{\psi_{\beta_{1}}(1) \otimes \psi_{\beta_{2}}(2)}\right]_{I \mu}^{T \tau}} \\
& =D_{\alpha} \hat{j_{1}} \hat{j}_{2} \sum_{\substack{L \Lambda S \Sigma \\
\lambda_{1} \lambda_{2}}}\left(\ell_{1} \lambda_{1} \ell_{2} \lambda_{2} \mid L \Lambda\right)(L \Lambda S \Sigma \mid I \mu) \\
& \quad \times \hat{L} \hat{S} \chi_{S \Sigma}(1,2) \chi_{T \tau}(1,2)\left[\psi_{\beta_{1}}^{\lambda_{1}}\left(\boldsymbol{r}_{1}\right) \psi_{\beta_{2}}^{\lambda_{2}}\left(\boldsymbol{r}_{2}\right)\right. \\
& \left.-(-)^{S+T} \psi_{\beta_{1}}^{\lambda_{1}}\left(\boldsymbol{r}_{2}\right) \psi_{\beta_{2}}^{\lambda_{2}}\left(\boldsymbol{r}_{1}\right)\right]\left\{\begin{array}{ccc}
\ell_{1} & s & j_{1} \\
\ell_{2} & s & j_{2} \\
L & S & I
\end{array}\right\},
\end{aligned}
$$

with $D_{\alpha}=1 / \sqrt{2\left(1+\delta_{\beta_{1} \beta_{2}}\right)}$ and where the angular momentum and isospin couplings used are summarized in Fig. 1. The nucleon-wave functions $\psi_{\beta}^{\lambda}\left(\boldsymbol{r}_{i}\right)$ are

$$
\psi_{\beta}^{\lambda}\left(\boldsymbol{r}_{i}\right)=u_{\beta}\left(r_{i}\right) Y_{\ell \lambda}\left(\hat{\boldsymbol{r}}_{i}\right)
$$

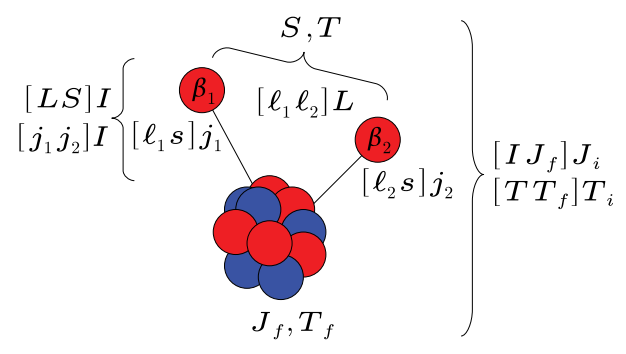

FIG. 1. (Color online) Angular momentum and isospin coupling schemes used in the calculations. The projectile initial (ground) state and final residue state have spin $J_{i}$ (projection $M_{i}$ ) and $J_{f}$ (projection $M_{f}$ ) and isospins $T_{i}$ (projection $\tau_{i}$ ) and $T_{f}$ (projection $\tau_{f}$ ), respectively. Each two-nucleon configuration $\alpha$ involves of a pair of active orbitals $\beta_{1}$ and $\beta_{2}$ with spherical shell-model quantum numbers $n_{i}, \ell_{i}$, and $j_{i}$ (projections $\lambda_{i}$ and $m_{i}$ ). In $L S$ coupling, the nucleon orbital angular momenta $\ell_{1}$ and $\ell_{2}$ are coupled to $L$ (projection $\Lambda$ ), the intrinsic spins to $S$ (projection $\Sigma$ ), and $L$ and $S$ to a total angular momentum $I$, which must also couple the initial and final-state total angular momenta.
It is convenient to combine the statistical factors and $9 j$ coefficient from the two-nucleon overlap with the appropriate $j j$-coupled TNAs to construct a set of $L S$-coupled TNAs, $\mathfrak{C}$, as

$$
\mathfrak{C}_{\alpha L S}^{I T}=\hat{j}_{1} \hat{j_{2}} \hat{L} \hat{S}\left\{\begin{array}{ccc}
\ell_{1} & s & j_{1} \\
\ell_{2} & s & j_{2} \\
L & S & I
\end{array}\right\} C_{\alpha}^{I T},
$$

that satisfy the sum rule

$$
\sum_{L S}\left[\mathfrak{C}_{\alpha L S}^{I T}\right]^{2}=\left[C_{\alpha}^{I T}\right]^{2}
$$

Antisymmetry requires, for configurations $\alpha$ where the nucleons originate from the same orbital, the $[n \ell j]^{2}$ cases, that $L+S+T$ is odd. For nucleons originating from different orbitals, this is no longer the case, though for two nucleons from spin-orbit partner orbitals, $\left[n \ell j_{<}, n \ell j_{>}\right]$with very similar radial wave functions, the $L+S+T=$ even amplitudes are also expected to be significantly suppressed.

\section{B. Eikonal model of two-nucleon stripping}

As was developed previously $[8,9,11]$, we exploit eikonal reaction dynamics. The elastic $S$-matrices describing the absorptive interactions of the $A$-body core (in state $f$ ) and the two nucleons with the target are calculated in the optical limit of Glauber's multiple scattering theory $[16,17]$ assuming that these projectile constituents travel on straight-line paths in the interaction field of the light target. The reaction is assumed to be sudden, such that the projectile internal co-ordinates are frozen on the time scale of this passing and interaction with the target. The eikonal $S$-matrices are calculated from the nucleonand heavy-residue-target interactions. These interactions were obtained by double folding the residue (core), nucleon ( $\delta$-function), and target-point particle densities with the usual effective nucleon-nucleon interaction, as used elsewhere, for example, Ref. [8].

Following Refs. $[8,9,11]$, from the total absorption cross section for the projectile-target system,

$$
\sigma_{\mathrm{abs}}=\frac{1}{\hat{J}_{i}^{2}} \sum_{M_{i}} \int d \boldsymbol{b}\left\langle\left.\Psi_{i}|1-| \mathcal{S}_{f} \mathcal{S}_{1} \mathcal{S}_{2}\right|^{2} \mid \Psi_{i}\right\rangle,
$$

which includes all events where one or more of the projectile constituents are absorbed by the target, we can identify and extract the two-nucleon stripping cross-section terms,

$$
\sigma_{\text {str }}=\frac{1}{\hat{J}_{i}^{2}} \sum_{M_{i}} \int d \boldsymbol{b}\left\langle\left.\Psi_{i}|| \mathcal{S}_{f}\right|^{2}\left(1-\left|\mathcal{S}_{1}\right|^{2}\right)\left(1-\left|\mathcal{S}_{2}\right|^{2}\right) \mid \Psi_{i}\right\rangle \text {. }
$$

As has been discussed elsewhere [11], the two-nucleon stripping probability $\mathcal{O}_{\text {str }}\left(b, b_{1}, b_{2}\right)=\left|\mathcal{S}_{f}\right|^{2}\left(1-\left|\mathcal{S}_{1}\right|^{2}\right)\left(1-\left|\mathcal{S}_{2}\right|^{2}\right)$ weights the impact parameters that contribute to these stripping events. Stripping requires an absorptive (inelastic) interaction of two nucleons with the target, but a non-absorptive (elastic) or non-interaction of the heavy residue with the target, and strongly localizes the reaction to grazing collisions at the projectile surface. This simplifies our picture of the reaction mechanism and of that part of the overlap function that is 
probed in the knockout reaction. We also contrast the present strong surface localization of the reaction with the term peripheral used by some authors to mean impact parameters that sample only the extreme tail (the Whittaker or Hankel function asymptotics) of the nucleon bound-state wave function. This is certainly not the case for those impact parameters selected by $\mathcal{O}_{\text {str }}\left(b, b_{1}, b_{2}\right)$ at the projectile energies and with the corresponding absorptive $S$-matrices of interest here.

Two further assumptions are made. The most important, which is supported by the stripping mechanism's selection of non-absorptive or non-interactive events of the residue and target, is to assume there is no dynamical excitation and/or change of state of the reaction residue by $\mathcal{S}_{f}$ in the collisionpreviously termed the spectator core approximation. This being the case,

$$
\left\langle\left.\Phi^{\left(F^{\prime}\right)}(A)|| \mathcal{S}_{f}\right|^{2} \mid \Phi^{(F)}(A)\right\rangle=\left|\mathcal{S}_{c}\right|^{2} \delta_{F F^{\prime}},
$$

where the bra and ket integrate out the internal coordinates of the residue and $\mathcal{S}_{c}$ is taken to be the residue ground-statetarget elastic scattering $S$-matrix. A lesser assumption is the heavy-core (no-recoil) approximation, which in the required integrals, such as Eq. (7), the residue impact parameter $b_{c}$ entering $\mathcal{S}_{f}$ can be replaced with that of the center of mass of the projectile $b$; that is, $b_{c} \approx b$. For the stripping terms, this approximation is not, in fact, needed, because a change of integration variable makes it unnecessary.

The result of these assumptions, together with the parentage expansion for the two-nucleon structure overlap Eq. (1), is that the exclusive stripping cross section to a given final-state $f$ can be written

$$
\begin{aligned}
\sigma_{\text {str }}^{(f)}= & \int d \boldsymbol{b}\left|\mathcal{S}_{c}\right|^{2} \frac{1}{\hat{J}_{i}^{2}} \sum_{M_{i} M_{f}}\left\langle\Psi_{i}^{(F)}\right|\left(1-\left|\mathcal{S}_{1}\right|^{2}\right) \\
& \times\left(1-\left|\mathcal{S}_{2}\right|^{2}\right)\left|\Psi_{i}^{(F)}\right\rangle .
\end{aligned}
$$

The assumption that the nucleon $S$-matrices are spin independent allows one to carry out all spin coordinate sums, in preparation for which we separate explicitly the nucleon position and spin variable integrations as

$$
\left\langle\Psi_{i}^{(F)}|\cdots| \Psi_{i}^{(F)}\right\rangle=\int d \boldsymbol{r}_{1} \int d \boldsymbol{r}_{2}\left\langle\Psi_{i}^{(F)}|\cdots| \Psi_{i}^{(F)}\right\rangle_{\mathrm{sp}},
$$

where the final bra-ket term denotes the spin integration.

Consideration of this (momentum-integrated) stripping cross section using $L S$ coupling was made in Ref. [8], with an emphasis on the reaction mechanism's lack of selectivity in the total spin $S$ of the two nucleons. In our previous analysis of the longitudinal momentum distributions [11] the only angular momentum projections not able to be summed over algebraically were those of the orbital angular momenta of the two nucleons.

With this observation made, we now consider the residue longitudinal momentum distributions in the $L S$ representation. The derivation follows a similar pattern to that in the $j j$-coupled algebra and begins from the $L S$-coupled, spin-integrated, modulus squared of the two-nucleon overlap, averaged over initial projections $M_{i}$ and summed over final projections $M_{f}$. Explicitly,

$$
\begin{aligned}
\frac{1}{\hat{J}_{i}^{2}} \sum_{M_{i} M_{f}}\left\langle\Psi_{i}^{(F)} \mid \Psi_{i}^{(F)}\right\rangle_{\mathrm{sp}} \\
=\frac{1}{\hat{J}_{i}^{2}} \sum_{\substack{I I^{\prime} \mu \mu^{\prime} \\
\alpha \alpha^{\prime} T T^{\prime}}} C_{\alpha}^{I T} C_{\alpha^{\prime}}^{I^{\prime} T^{\prime}} \sum_{M_{i} M_{f}}\left(I \mu J_{f} M_{f} \mid J_{i} M_{i}\right) \\
\quad \times\left(I^{\prime} \mu^{\prime} J_{f} M_{f} \mid J_{i} M_{i}\right)\left(T \tau T_{f} \tau_{f} \mid T_{i} \tau_{i}\right)\left(T^{\prime} \tau^{\prime} T_{f} \tau_{f} \mid T_{i} \tau_{i}\right) \\
\quad \times\left\langle\left[\overline{\psi_{j_{1}^{\prime}}(1) \otimes \psi_{j_{2}^{\prime}}(2)}\right]_{I^{\prime} \mu^{\prime}}^{T^{\prime} \tau^{\prime}} \mid\left[\overline{\psi_{j_{1}}(1) \otimes \psi_{j_{2}}(2)}\right]_{I \mu}^{T \tau}\right\rangle_{\mathrm{sp}} \cdot
\end{aligned}
$$

On performing the sums over $M_{i}$ and $M_{f}$ the expression is clearly incoherent in the coupled two-nucleon total angular momentum $I \mu$, a consequence of the spectator-core approximation Eq. (8). Using the antisymmetric two-nucleon $L S$ coupled forms of Eqs. (2) and (4), and assuming the nucleon $S$-matrices are also isospin-independent, we can perform the isospin sums with the result that Eq. (11) is also incoherent with respect to both $S$ and $T$. Finally, summing over the projections of $I$ and $S$ we obtain the result, incoherent also in $L$ and $\Lambda$, namely,

$$
\begin{aligned}
\frac{1}{\hat{J}_{i}^{2}} \sum_{M_{i} M_{f}}\left\langle\Psi_{i}^{(F)} \mid \Psi_{i}^{(F)}\right\rangle_{\mathrm{sp}} \\
=\sum_{T}\left(T \tau T_{f} \tau_{f} \mid T_{i} \tau_{i}\right)^{2} \sum_{I L S \alpha \alpha^{\prime}} \frac{\mathfrak{C}_{\alpha L S}^{I T} \mathfrak{C}_{\alpha^{\prime} L S}^{I T} D_{\alpha} D_{\alpha^{\prime}}}{\hat{L}^{2}} \\
\quad \times \sum_{\Lambda \lambda_{1} \lambda_{2} \lambda_{1}^{\prime} \lambda_{2}^{\prime}}\left(\ell_{1} \lambda_{1} \ell_{2} \lambda_{2} \mid L \Lambda\right)\left(\ell_{1}^{\prime} \lambda_{1}^{\prime} \ell_{2}^{\prime} \lambda_{2}^{\prime} \mid L \Lambda\right) \\
\quad \times\left[\psi_{\beta_{1}^{\prime}}^{\lambda_{1}^{\prime}}\left(\boldsymbol{r}_{1}\right) \psi_{\beta_{2}^{\prime}}^{\lambda_{2}^{\prime}}\left(\boldsymbol{r}_{2}\right)-(-)^{S+T} \psi_{\beta_{1}^{\prime}}^{\lambda_{1}^{\prime}}\left(\boldsymbol{r}_{2}\right) \psi_{\beta_{2}^{\prime}}^{\lambda_{2}^{\prime}}\left(\boldsymbol{r}_{1}\right)\right]^{*} \\
\quad \times\left[\psi_{\beta_{1}}^{\lambda_{1}}\left(\boldsymbol{r}_{1}\right) \psi_{\beta_{2}}^{\lambda_{2}}\left(\boldsymbol{r}_{2}\right)-(-)^{S+T} \psi_{\beta_{1}}^{\lambda_{1}}\left(\boldsymbol{r}_{2}\right) \psi_{\beta_{2}}^{\lambda_{2}}\left(\boldsymbol{r}_{1}\right)\right] .
\end{aligned}
$$

The exclusive two-nucleon stripping cross section is then given by use of this structure overlap information in Eq. (9). In the following we derive explicit expressions for the associated exclusive momentum distributions in this $L S$ representation.

\section{Residue momentum distributions}

Structurally, the expressions for the residue momentum distributions in the $L S$-coupling scheme are similar to those using $j j$ coupling [11]. The derivations also follow a largely parallel procedure. The coordinate system used is reproduced in Fig. 2 for clarity of the following expressions.

The reaction samples the momentum content of the bound-state wave functions of the stripped nucleons in the direction of the projectile beam $\hat{\boldsymbol{k}}$ (i.e., the $z$ axis). For fixed values of the $s_{i}$, and hence fixed nucleon impact parameters $b_{i}=\left|\boldsymbol{b}+\boldsymbol{s}_{i}\right|, \quad i=1,2$, this information is carried by the functions

$$
\mathcal{R}_{\beta}^{\lambda}(i)=\frac{\mathcal{C}_{\ell \lambda}}{\sqrt{2 \pi}} \int_{-\infty}^{+\infty} d z_{i} u_{\beta}\left(r_{i}\right) P_{\ell}^{|\lambda|}\left(\cos \theta_{i}\right) \exp \left[i \kappa_{i} z_{i}\right]
$$

where $\kappa_{i}$ is the $z$ component of the momentum of nucleon $i$ in the projectile's rest frame. We note that the notation for $\mathcal{R}_{\beta}^{\lambda}(i)$ is changed from that of Ref. [11] consistent with the notation used 


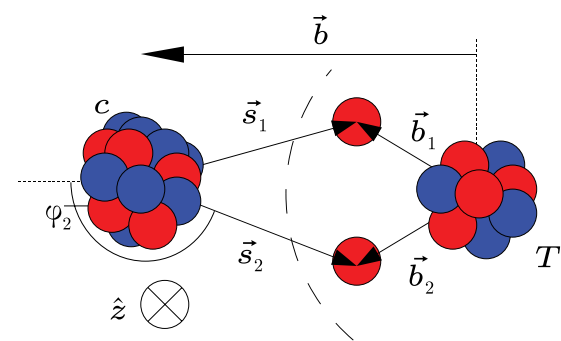

FIG. 2. (Color online) Schematic of the particle coordinates used. Vectors $s_{i}$ are the components, in the plane perpendicular to the beam direction (the $z$ axis), of the position vectors $\boldsymbol{r}_{i}$ of the knocked-out nucleons relative to the core of nucleons to which they are initially bound. The two nucleons have impact parameters $\boldsymbol{b}_{\boldsymbol{i}}=\boldsymbol{b}+\boldsymbol{s}_{i}$ relative to the target nucleus.

for the $u_{\beta}\left(r_{i}\right)$. The correct weighting of the nucleon absorption probability with the azimuthal angle $\varphi_{i}$, of $\boldsymbol{s}_{i}$, is carried by the functions $\mathcal{H}_{\lambda \lambda^{\prime}}(i) \equiv \mathcal{H}_{\lambda \lambda^{\prime}}\left(b, \boldsymbol{s}_{i}\right)$, given by

$$
\mathcal{H}_{\lambda \lambda^{\prime}}(i)=\int_{0}^{2 \pi} d \varphi_{i}\left[1-\left|\mathcal{S}_{i}\left(\left|\boldsymbol{b}+\boldsymbol{s}_{i}\right|\right)\right|^{2}\right] \exp \left[i \varphi_{i}\left(\lambda-\lambda^{\prime}\right)\right],
$$

with $\boldsymbol{s}_{i}$ the components of the $\boldsymbol{r}_{i}$ in the impact-parameter plane; that is, $\boldsymbol{r}_{i}=\boldsymbol{s}_{i}+z_{i} \hat{\boldsymbol{k}}$. The remaining details of the derivation are completely analogous to those in Ref. [11], to which the reader is referred.

We obtain the projectile rest frame, stripping mechanism momentum distribution as the incoherent $L S$, and isospin decomposition

$$
\begin{aligned}
\frac{d \sigma_{\mathrm{str}}^{(f)}}{d \kappa_{c}}= & \sum_{L S T} \frac{d \sigma_{L S T}^{(f)}}{d \kappa_{c}} \\
= & \sum_{T}\left(T \tau T_{f} \tau_{f} \mid T_{i} \tau_{i}\right)^{2} \sum_{L S I \alpha \alpha^{\prime}} \frac{2 \mathfrak{C}_{\alpha L S}^{I T} \mathfrak{C}_{\alpha^{\prime} L S}^{I T} D_{\alpha} D_{\alpha^{\prime}}}{\hat{L}^{2}} \\
& \times \int d \kappa_{1} \int d \kappa_{2} \delta\left(\kappa_{c}+\kappa_{1}+\kappa_{2}\right) \int d \boldsymbol{b}\left|\mathcal{S}_{c}(b)\right|^{2} \\
& \times \sum_{\Lambda \lambda_{1} \lambda_{2} \lambda_{1}^{\prime} \lambda_{2}^{\prime}}\left(\ell_{1} \lambda_{1} \ell_{2} \lambda_{2} \mid L \Lambda\right)\left(\ell_{1}^{\prime} \lambda_{1}^{\prime} \ell_{2}^{\prime} \lambda_{2}^{\prime} \mid L \Lambda\right) \\
& \times \int d s_{1} s_{1} \int d s_{2} s_{2}[\text { direct - exchange], }
\end{aligned}
$$

where the direct term is

$$
\operatorname{direct}=\left\{\mathcal{H}_{\lambda_{1} \lambda_{1}^{\prime}}(1) \mathcal{R}_{\beta_{1}}^{\lambda_{1}}(1) \mathcal{R}_{\beta_{1}^{\prime}}^{\lambda_{1}^{\prime}}(1)^{*} \mathcal{H}_{\lambda_{2} \lambda_{2}^{\prime}}(2) \mathcal{R}_{\beta_{2}}^{\lambda_{2}}(2) \mathcal{R}_{\beta_{2}^{\prime}}^{\lambda_{2}^{\prime}}(2)^{*}\right\},
$$

and the exchange term,

$$
\begin{aligned}
\text { exchange }= & (-1)^{S+T}\left\{\mathcal{H}_{\lambda_{2} \lambda_{1}^{\prime}}(1) \mathcal{R}_{\beta_{2}}^{\lambda_{2}}(1) \mathcal{R}_{\beta_{1}^{\prime}}^{\lambda_{1}^{\prime}}(1)^{*}\right. \\
& \left.\times \mathcal{H}_{\lambda_{1} \lambda_{2}^{\prime}}(2) \mathcal{R}_{\beta_{1}}^{\lambda_{1}}(2) \mathcal{R}_{\beta_{2}^{\prime}}^{\lambda_{2}^{\prime}}(2)^{*}\right\} .
\end{aligned}
$$

It should be noted that Eq. (15) (which contains a factor of 2) and these simplified forms for the direct and exchange terms compared to those in Ref. [11] assume that the integrals over both the $\kappa_{i}$ and the $s_{i}$ will be carried out, and so one is computing quantities that are completely symmetric in the two-nucleon coordinates. Also, unlike for the $j j$-coupled scheme, no further recoupling is required to reduce the angular momentum algebra.

Physically, Eq. (15) shows that the sums of the direct and exchange terms over the $\lambda$ are independent of $I$. The resulting momentum distributions thus depend explicitly on $L$ (and $S+T$ via the phase of the exchange term), but not on $I$. We see that the significance of $I$ and the nucleon total angular momenta $j_{i}$ is that they will determine the relative strengths of the different $L$ and $S$ via the amplitudes $\mathfrak{C}_{\alpha L S}^{I T}$. Thus, it is $L$, and to a lesser extent $S$ and $T$, that will determine the shape of the residue's momentum distribution. $I$, however, will be important in determining the relative strengths of the $L$ and $S$ that contribute.

\section{TWO-NUCLEON CORRELATIONS}

We observe that the expression for residue momentum distributions is somewhat simpler when using $L S$ coupling, having a more transparent angular momentum dependence. However, the dependence on the two-nucleon configurations, via $\alpha$ (and $\alpha^{\prime}$ ), is still less than transparent in Eq. (15). We attempt to elucidate this important nuclear structure sensitivity by carrying out the $\lambda$ projection sums. Before doing so we introduce and discuss the two-nucleon joint position probability that summarizes both the strength and the spatial localization (and correlation) of the two nucleons in the structure overlaps that affect the stripping yield.

\section{A. Two-nucleon joint position probability}

We consider the two-nucleon joint position probability relevant to the removal reaction transition to a given final state $f$; that is,

$$
\rho_{f}\left(\boldsymbol{r}_{1}, \boldsymbol{r}_{2}\right)=\frac{1}{\hat{J}_{i}^{2}} \sum_{M_{i} M_{f}}\left\langle\Psi_{i}^{(F)} \mid \Psi_{i}^{(F)}\right\rangle_{\mathrm{sp}} .
$$

While the production of a given residue final state by the two-nucleon knockout mechanism will depend on the details of $\rho_{f}\left(\boldsymbol{r}_{1}, \boldsymbol{r}_{2}\right)$, specifically, the extent to which there is a spatial proximity of the two nucleons at the projectile surface, its overall normalization, and the $L S$ composition of this normalization,

$$
\begin{aligned}
N_{f} & =\int d \boldsymbol{r}_{1} \int d \boldsymbol{r}_{2} \rho_{f}\left(\boldsymbol{r}_{1}, \boldsymbol{r}_{2}\right) \\
& =\sum_{\alpha I T}\left[\left(T \tau T_{f} \tau_{f} \mid T_{i} \tau_{i}\right) C_{\alpha}^{I T}\right]^{2} \\
& =\sum_{L S}\left\{\sum_{\alpha I T}\left[\left(T \tau T_{f} \tau_{f} \mid T_{i} \tau_{i}\right) \mathfrak{C}_{\alpha L S}^{I T}\right]^{2}\right\}=\sum_{L S} N_{f}^{L S},
\end{aligned}
$$

are measures of the likely transition strength. In the case of a single (dominant) two-nucleon structure configuration this $L S$ breakdown can also guide the relative strengths expected from the different contributing $L S$ terms for a given final state. However, when configurations are mixed or where the initial and final states have different parity, interference effects may strongly affect these relative strengths. 
Because the projectile is assumed to traverse a straight-line path in the $z$ direction, it is useful for what follows, and also highly intuitive, to construct the projection of the two-nucleon joint position probability onto the impact-parameter planethe plane perpendicular to the beam direction - by integrating over the $z_{i}$ of the two nucleons,

$$
\mathcal{P}_{f}\left(\boldsymbol{s}_{1}, \boldsymbol{s}_{2}\right)=\int d z_{1} \int d z_{2} \rho_{f}\left(\boldsymbol{r}_{1}, \boldsymbol{r}_{2}\right) .
$$

The relevant spatial correlation for the reaction is now the degree of localization of the probability with respect to the two-nucleon coordinate projections $s_{i}$ in this impact parameter plane.

In what follows the correlation of the two nucleons is concisely expressed as a function of the angular separation, $\omega$, of their position coordinates $\boldsymbol{r}_{i}$. Clearly, the $z_{i}$-integrated joint probability $\mathcal{P}_{f}\left(\boldsymbol{s}_{1}, \boldsymbol{s}_{2}\right)$ will see a smeared version of this correlation function because fixed $s_{i}$ will sample a range of $\omega$. However, because the reaction is surface localized and the target is light (small), the effective thickness in the $z_{i}$ will tend to be rather restricted and $\mathcal{P}_{f}\left(\boldsymbol{s}_{1}, \boldsymbol{s}_{2}\right)$ will remain a useful construct and intuitive link to the magnitudes of the two-nucleon knockout cross sections.

As was indicated by Figs. 2 and 3 of Ref. [18], and is emphasized here, the total angular momentum of the final state and the detailed TNAs of the wave function can strongly affect the two-nucleon joint position probability, its projection, and the magnitude of the removal cross sections. The (shell-model) structural correlations may also enhance or suppress particular total orbital angular momenta and so may affect the residue momentum distributions also.

\section{B. Angular correlations}

Despite the relative simplifications introduced by $L S$ coupling, the momentum distribution expression Eq. (15) remains a complicated weighted sum of wave-function transforms. Moreover, it still depends on the orbital angular momentum projections. To clarify the underlying sensitivity to twonucleon correlations, we simplify the spin-integrated twonucleon joint position probability of Eq. (18) by summing out the $\lambda$ projection labels.

The relevant terms we need to simplify are, for the direct terms of Eq. (12),

$$
\begin{aligned}
\Gamma_{\ell_{1} \ell_{2} \ell_{1}^{\prime} \ell_{2}^{\prime}}^{L}\left(\hat{\boldsymbol{r}}_{1}, \hat{\boldsymbol{r}}_{2}\right)= & \sum_{\Lambda \lambda_{1} \lambda_{2} \lambda_{1}^{\prime} \lambda_{2}^{\prime}}\left(\ell_{1} \lambda_{1} \ell_{2} \lambda_{2} \mid L \Lambda\right)\left(\ell_{1}^{\prime} \lambda_{1}^{\prime} \ell_{2}^{\prime} \lambda_{2}^{\prime} \mid L \Lambda\right) \\
& \times Y_{\ell_{1} \lambda_{1}}\left(\hat{\boldsymbol{r}}_{1}\right) Y_{\ell_{1}^{\prime} \lambda_{1}^{\prime}}^{*}\left(\hat{\boldsymbol{r}}_{1}\right) Y_{\ell_{2} \lambda_{2}}\left(\hat{\boldsymbol{r}}_{2}\right) Y_{\ell_{2}^{\prime} \lambda_{2}^{\prime}}^{*}\left(\hat{\boldsymbol{r}}_{2}\right) .
\end{aligned}
$$

Combining the spherical harmonics of the same argument, summing the $\lambda$ projections, and using the spherical harmonics addition theorem, one obtains

$$
\begin{aligned}
\Gamma_{\ell_{1} \ell_{2} \ell_{1}^{\prime} \ell_{2}^{\prime}}^{L}(\omega)= & (-1)^{L} \frac{\hat{\ell}_{1} \hat{\ell}_{1}^{\prime} \hat{\ell}_{2} \hat{\ell}_{2}^{\prime} \hat{L}^{2}}{(4 \pi)^{2}} \sum_{k} W\left(\ell_{1} \ell_{2} \ell_{1}^{\prime} \ell_{2}^{\prime} ; L k\right) \\
& \times(-1)^{k}\left(\ell_{1} 0 \ell_{1}^{\prime} 0 \mid k 0\right)\left(\ell_{2} 0 \ell_{2}^{\prime} 0 \mid k 0\right) P_{k}(\cos \omega),
\end{aligned}
$$

where $\omega$ is the angular separation of the two nucleons; that is, $\cos \omega=\boldsymbol{r}_{1} \cdot \boldsymbol{r}_{2} / r_{1} r_{2}$. A similar result can be found in Ref. [19].

The angular correlation function $\Gamma_{\ell_{1} \ell_{2} \ell_{1}^{\prime} \ell_{2}^{\prime}}^{L}(\omega)$ is seen to be independent of the total angular momentum $I$ and of the individual angular momenta $j_{i}$ of the nucleons. However, it depends explicitly on their orbital angular momenta and on the total orbital angular momentum $L$. The form written previously is that for the direct terms of Eq. (12). The exchange terms differ by a phase owing to the reordering of the angular momentum labels in the exchange form of Eq. (21), as is given in what follows.

The radial behaviors associated with the direct and exchange terms of the joint-probability density are

$$
\begin{aligned}
U_{\alpha \alpha^{\prime}}^{D}\left(r_{1}, r_{2}\right)= & u_{\beta_{1}}\left(r_{1}\right) u_{\beta_{2}}\left(r_{2}\right) u_{\beta_{1}^{\prime}}\left(r_{1}\right) u_{\beta_{2}^{\prime}}\left(r_{2}\right) \\
& +u_{\beta_{2}}\left(r_{1}\right) u_{\beta_{1}}\left(r_{2}\right) u_{\beta_{2}^{\prime}}\left(r_{1}\right) u_{\beta_{1}^{\prime}}\left(r_{2}\right), \\
U_{\alpha \alpha^{\prime}}^{E}\left(r_{1}, r_{2}\right)= & u_{\beta_{1}}\left(r_{1}\right) u_{\beta_{2}}\left(r_{2}\right) u_{\beta_{2}^{\prime}}\left(r_{1}\right) u_{\beta_{1}^{\prime}}\left(r_{2}\right) \\
& +u_{\beta_{2}}\left(r_{1}\right) u_{\beta_{1}}\left(r_{2}\right) u_{\beta_{1}^{\prime}}\left(r_{1}\right) u_{\beta_{2}^{\prime}}\left(r_{2}\right) .
\end{aligned}
$$

In terms of these and the corresponding direct and exchange angular correlation functions, the two-nucleon joint-probability density is

$$
\begin{aligned}
& \rho_{f}\left(\boldsymbol{r}_{1}, \boldsymbol{r}_{2}\right) \\
& =\sum_{L S T} \sum_{I \alpha \alpha^{\prime}} \frac{\mathfrak{C}_{\alpha L S}^{I T} \mathfrak{C}_{\alpha^{\prime} L S}^{I T} D_{\alpha} D_{\alpha^{\prime}}}{\hat{L}^{2}}\left(T \tau T_{f} \tau_{f} \mid T_{i} \tau_{i}\right)^{2} \\
& \quad \times\left[U_{\alpha \alpha^{\prime}}^{D}\left(r_{1}, r_{2}\right) \Gamma^{L, D}(\omega)-(-)^{S+T} U_{\alpha \alpha^{\prime}}^{E}\left(r_{1}, r_{2}\right) \Gamma^{L, E}(\omega)\right],
\end{aligned}
$$

with $\Gamma^{L, D}(\omega) \equiv \Gamma_{\ell_{1} \ell_{2} \ell_{1}^{\prime} \ell_{2}^{\prime}}^{L}(\omega)$ given by Eq. (22) and

$$
\Gamma^{L, E}(\omega)=(-)^{\ell_{1}^{\prime}+\ell_{2}^{\prime}-L} \Gamma_{\ell_{1} \ell_{2} \ell_{2}^{\prime} \ell_{1}^{\prime}}^{L}(\omega)
$$

It is clear, therefore, that the angular correlation function dictates how the spatial correlations change with angular momentum coupling and that $L$ is crucial, the $U\left(r_{1}, r_{2}\right)$ being dependent on the $\beta_{i}$ but independent of the angular momentum coupling. Clear also is that, in cases where the radial wave functions for all active orbits are similar, the angular correlation function alone will determine the differences in residue momentum distributions for the different possible angular momentum couplings. As discussed earlier, these differences, generated at the angular correlation function and the two-nucleon density level, are more distinct than in the projected density Eq. (20), where fixed co-ordinate pairs $\left(\boldsymbol{s}_{1}, \boldsymbol{s}_{2}\right)$ sample a range of angular separations $\omega$ and so smear the spatial correlations.

Uncorrelated two-nucleon models, discussed in the Introduction and Refs. [6,8], that neglect antisymmetrization, angular momentum coupling, and parentage coefficients, lead to a constant, $\omega$-independent correlation function. For twonucleon removal from a single $\left[s_{1 / 2}\right]^{2}$ configuration, the angular correlation function is also seen to be $\omega$ independent $(k=0)$ and the uncorrelated (see Ref. [11]) and fully correlated residue momentum distributions are identical. 


\section{Cross-shell excitations}

Here we consider briefly the implications for two-nucleon knockout from configurations with $\beta_{1}$ and $\beta_{2}$ of different parity. It is well established that the addition of shell-model configurations with $1 \hbar \omega, 3 \hbar \omega$ single-particle excitations is required to obtain a high degree of surface pairing (see, e.g., Refs. [20-25]).

We obtain a similar result here by considering the symmetry of the angular correlation function about $\omega=\pi / 2$. In $\Gamma_{\ell_{1} \ell_{2} \ell_{1}^{\prime} \ell_{2}^{\prime}}^{L}(\omega)$, only the Legendre polynomial depends on $\omega$, with the property that $P_{k}(\cos [\pi-\omega])=(-1)^{k} P_{k}(\cos \omega)$. Because the values of $k$ are restricted to be odd or even by the parity Clebsch Gordan coefficients, the angular correlation will be even about $\pi / 2$ for $\pi_{\ell} \pi_{\ell^{\prime}}=+1$ and odd about $\pi / 2$ for $\pi_{\ell} \pi_{\ell^{\prime}}=-1$. In the absence of single-particle excitations of the kind $1 \hbar \omega$, the probability for finding the nucleon pair with angular separation $\omega=0$ and $\pi$ are equal and a high degree of two-nucleon pair cluster structure will not be obtained.

So pair correlations will be enhanced in cases when there is mixing between two-nucleon configurations where the orbital angular momenta are of different parity. Whether the interference is constructive or destructive will depend on the sign of $\Gamma_{\ell_{1} \ell_{2} \ell_{1}^{\prime} \ell_{2}^{\prime}}^{L}(\omega)$ near $\omega=0$, the relative signs of the $9 j$ coefficients, and the relative signs of the TNAs. A specific two-configuration example is presented in Sec. IV C.

These results are quite general in that they do not depend on the pair total angular momentum $I$; enhancements in the spatial correlations in the two-nucleon density may be found for $I \neq 0$.

\section{ILLUSTRATIVE EXAMPLES}

Previous calculations of exclusive two-nucleon removal residue momentum distributions noted a strong sensitivity to the total angular momentum of the removed nucleon pair. Here, by writing this momentum-differential cross section in $L S$ coupling, and by a consideration of the angular correlations inherent in the two-nucleon joint probability function, it becomes apparent that the crucial sensitivity of this observable is to the total orbital angular momentum values, $L$, contributing to the transition. These different $L$ components will contribute incoherently to the cross section yields and their momentum distributions. These theoretical observations and the resulting sensitivity of the momentum distribution observable offer the potential to probe more subtle features of the nucleon pair's configurations and the correlations present in the shell-model wave functions used.

A generic first example arises if the predominant two-nucleon configuration populating a given final state involves one of the nucleons in an $s$-wave orbital. In this case the total orbital angular momentum is restricted to the orbital angular momentum of the second active orbit, $L=\ell$, and thus $L$ is pure. It is expected, therefore, that there can be distinct differences in the momentum distributions, even for states of the same total angular momentum $I$, for example, for two $3^{+}$final states built from $\left[g_{7 / 2}, s_{1 / 2}\right]$ and from $\left[d_{5 / 2}, s_{1 / 2}\right]$. More generally, even where there is significant mixing and several active configurations, the structure of specific states in the spectrum can be rather $L$ pure. So the reaction will proceed by a particular $L$ with a momentum distribution that is characteristic of this structure.

In the following we discuss specific examples from different $A$ and $Z$ regions of the nuclear chart. In each example the nucleon bound-state radial wave functions required for the two-nucleon overlaps are calculated using a Woods-Saxon potential well with a spin-orbit term of depth $6 \mathrm{MeV}$ and a diffuseness parameter $a_{0}=0.7 \mathrm{fm}$. Unless stated otherwise, the geometries (the radius parameters $r_{0}$ ) of the potential wells in each case were adjusted to reproduce the root-mean-square radii and the separation energies of spherical Hartree-Fock calculations using the Skyrme (SkX) interaction parametrization [26] for the active orbitals in question. The specific procedure was detailed in Ref. [5]. These fitted geometries are then used to calculate the radial wave functions needed using the empirical, effective nucleon-separation energies. Where required, shell-model calculations are performed using the code OXBASH [27]. The model spaces and interactions used are specified for each case studied in what follows.

\section{A. $p$-shell example: ${ }^{12} \mathrm{C}(-n p)$}

Here we consider the removal of a $(T=0,1)$ neutron and proton $(n p)$ pair from ${ }^{12} \mathrm{C}$ at $2100 \mathrm{MeV} /$ nucleon on a ${ }^{12} \mathrm{C}$ target. The proton and neutron orbits are taken to be identical with radial wave functions calculated in a Woods-Saxon potential, using an average nucleon charge $\bar{Z}=0.5$. The geometry of the Woods-Saxon potential was fixed with $r_{0}=$ $1.31 \mathrm{fm}, a_{0}=0.55 \mathrm{fm}$. Both the ${ }^{10} \mathrm{~B}$ residue and the ${ }^{12} \mathrm{C}$ target were assumed have Gaussian-shaped mass distributions, with rms radii 2.30 and $2.32 \mathrm{fm}$, respectively. The isospin format TNAs are calculated using OXBASH in a $p$-shell model space using the WBP interaction [28], as in previous studies [29,30]. A more complete consideration of two-nucleon removal from ${ }^{12} \mathrm{C}$ will be discussed in a forthcoming article.

As a specific example, we consider the first and second $T=0,{ }^{10} \mathrm{~B}\left(1^{+}\right)$final states. The TNAs for these states are shown in Table I. The relative magnitudes of the contributing two-nucleon configurations to these states are different and it is of interest to consider how these differences might affect the cross sections and their momentum distributions. The sum of the squared TNAs for the first and second states are 1.45 and 1.47, respectively; thus, in the absence of interference terms the incoherent sum of contributions from each of these configurations would yield very similar cross sections.

That this is not the case is shown by the calculated two-nucleon stripping cross sections presented in Table II. The calculated momentum distributions are also rather different, as is shown in Fig. 3.

TABLE I. Isospin format TNAs for the first and second ${ }^{10} \mathrm{~B}\left(1^{+}\right)$ $T=0$ states populated by neutron-proton removal from ${ }^{12} \mathrm{C}$. The relative strengths of the TNAs are different for the two states.

\begin{tabular}{lrcr}
\hline \hline$J_{f}^{\pi}$ & {$\left[1 p_{3 / 2}\right]^{2}$} & {$\left[1 p_{1 / 2}, 1 p_{3 / 2}\right]$} & \multicolumn{1}{c}{$\left[1 p_{1 / 2}\right]^{2}$} \\
\hline $1_{1}^{+}$ & 0.69899 & 0.97868 & -0.01067 \\
$1_{2}^{+}$ & -1.13385 & 0.22886 & 0.36314 \\
\hline \hline
\end{tabular}


TABLE II. Two-nucleon stripping cross sections $\sigma_{L S}$ for populating the first two $T=0, J_{f}=1^{+}$final states in ${ }^{10} \mathrm{~B}$ for a ${ }^{12} \mathrm{C}$ beam energy of $2100 \mathrm{MeV}$ per nucleon. All cross sections are in mb.

\begin{tabular}{cccccc}
\hline \hline$J_{f}^{\pi}$ & $\sigma_{01}$ & $\sigma_{10}$ & $\sigma_{11}$ & $\sigma_{21}$ & $\sigma_{\text {str }}$ \\
\hline $1_{1}^{+}$ & 2.41 & 0.00 & 0.00 & 0.06 & 2.47 \\
$1_{2}^{+}$ & 0.60 & 0.59 & 0.00 & 0.63 & 1.81 \\
\hline \hline
\end{tabular}

These differences can be understood by reference to the projected two-particle joint position probabilities for the two states, which are strikingly different (see Fig. 4). The first $1^{+}$state shows strong spatial localization of the two nucleons, favorable for the two-nucleon removal cross section. Both example position probabilities manifest the expected symmetry about a nucleon angular separation of $\phi_{12}=\pi / 2$, because the model space is restricted to the $p$ shell and the active orbitals have the same parity.

We can extend this $p$-shell example further to illustrate the potential for large sensitivity to the underlying structure. It is clear from Eq. (24) that within a $p$-shell model space the relative strengths of different $L S$ combinations are determined solely by the TNAs and the nucleon configurations involved. So, neglecting any minor differences in the $p$-wave radial wave functions, owing to spin-orbit splitting, the entire square bracketed term in Eq. (24) is independent of the total angular momenta $\left\{j_{i}\right\}$ and, in the present model space, independent of the configurations $\left(\alpha, \alpha^{\prime}\right)$ of the pair. It follows that the weight of each $L$ and $S$ term in a state of given $I$ and $T$ is proportional to

$$
P_{L S}^{I T}=\sum_{\alpha \alpha^{\prime}} \mathfrak{C}_{\alpha L S}^{I T} \mathfrak{C}_{\alpha^{\prime} L S}^{I T} D_{\alpha} D_{\alpha^{\prime}}
$$

In the sprit of studying the extremes of possible sensitivity of the momentum distributions, we may force any one of these

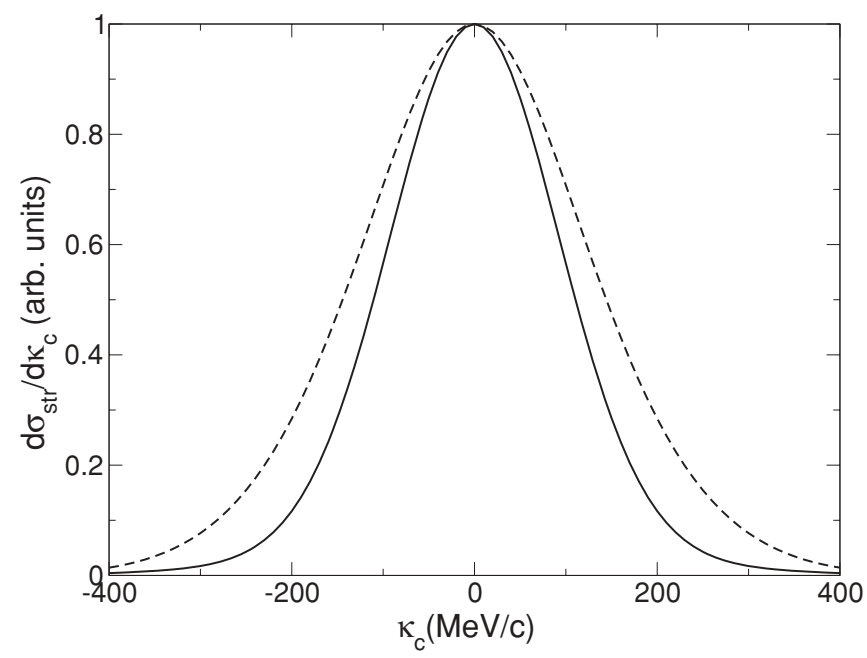

FIG. 3. Normalized residue momentum distributions for the first (solid) and second (dashed) ${ }^{10} \mathrm{~B}\left(J_{f}=1^{+}\right)$states populated in $n p$ knockout from ${ }^{12} \mathrm{C}$ at $2100 \mathrm{MeV}$ per nucleon. Though the same two-nucleon configurations contribute to each state, the differently weighted TNAs result in distinct momentum distributions.

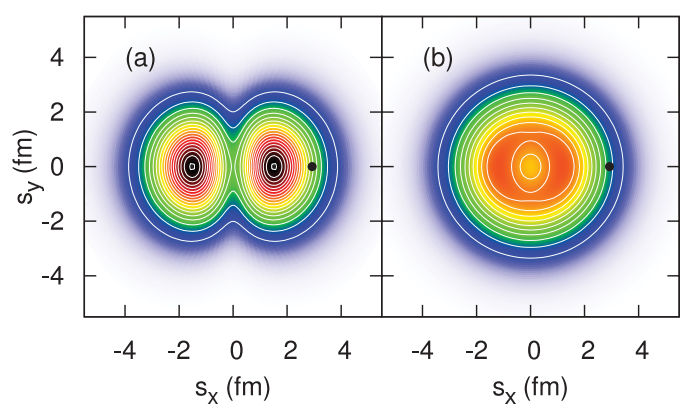

FIG. 4. (Color online) Impact-parameter-plane-projected joint position probabilities for (a) the first and (b) second $T=0{ }^{10} \mathrm{~B}\left(1^{+}\right)$ states populated via $n p$ knockout from ${ }^{12} \mathrm{C}$. The plot shows the impactparameter-plane probability distribution of nucleon 2 for nucleon 1 positioned at $s_{x}=2.9 \mathrm{fm}, s_{y}=0 \mathrm{fm}$. The spatial correlations of the nucleon pairs in these two states are fundamentally different, leading to markedly different momentum distributions (see Fig. 3). The color scale (white-blue-green-yellow-red-black) is common to both plots.

$P_{L S}^{I T}$ to be zero and solve for the relative strengths and phases of the $\mathfrak{C}_{\alpha L S}^{I T}$ and $C_{\alpha}^{I T}$ needed to achieve this.

Figure 5 illustrates such examples for assumed $I=1$, $T=0$ states populated via the configurations $\alpha_{1}=\left[1 p_{1 / 2}\right]^{2}$ and $\alpha_{2}=\left[1 p_{3 / 2}, 1 p_{1 / 2}\right]$. Calculations for two sets of TNAs are shown: one set chosen to eliminate $L=0, S=1$ contributions (requiring $C_{\alpha_{1}}^{10}=-4 C_{\alpha_{2}}^{10}$, dashed curve) and the other to eliminate $L=2, S=1$ contributions (requiring $C_{\alpha_{1}}^{10}=C_{\alpha_{2}}^{10} / 2$, solid curve). These different $1^{+}$state TNAs produce wide and narrow residue momentum distributions, respectively, the difference in the FWHM widths being almost a factor of two. The figure also shows the $I=2, T=1$ momentum distribution (open circles), populated via $\alpha_{2}=\left[1 p_{3 / 2}, 1 p_{1 / 2}\right]$ and $\alpha_{3}=\left[1 p_{3 / 2}\right]^{2}$, where the TNAs were chosen to eliminate

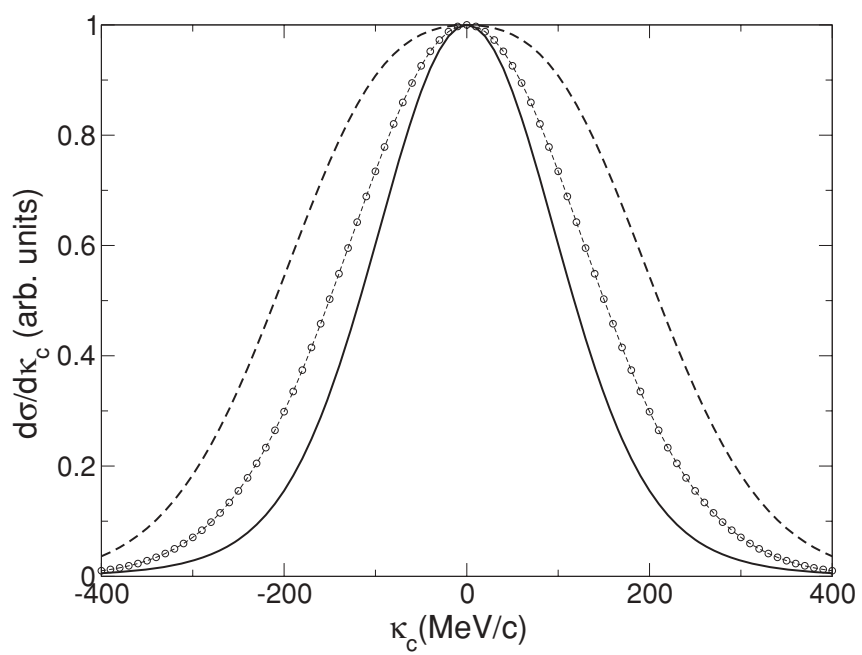

FIG. 5. Theoretical ${ }^{10} \mathrm{~B}$ residue momentum distributions at $2100 \mathrm{MeV}$ per nucleon. Shown are the expectations for two $I=1$ $T=0$ states where the TNAs have been tailored to exclude $L=$ 2, $S=1$ (solid line) and $L=0, S=1$ (dashed line) contributions from the two-nucleon wave function, respectively. The line with open circles shows the expectation for an $I=2 T=1$ final state where the TNAs were similarly chosen to eliminate $L=2, S=1$ contributions. 
$L=2, S=1$ contributions (requiring $C_{\alpha_{3}}^{21}=-\sqrt{2} C_{\alpha_{2}}^{21}$ ). Once again, this gives a relatively narrow distribution and, moreover, this $I=2$ distribution is narrower than that for the $(L=0$ excluded) $I=1$ distribution (dashed curve) described earlier. These examples break the tie between the width of the momentum distribution and the $I$ value of the transferred pair. Whether or not nuclear states with these TNAs are physically realized, these limiting cases demonstrate how details of the microscopic structure of a given state may strongly influence the shapes and widths of the expected residue momentum distributions.

Similarly, we note the expectation that transitions to $I=2, T=0$ and $I=3, T=0$ states of ${ }^{10} \mathrm{~B}$ will, within a $p$-shell model space, yield identical theoretical momentum distributions because both transitions are pure $L=2$ in nature. In this instance, also the width of the momentum distribution does not provide a direct measure of $J_{f}=I$.

We note that consideration has only been given to the direct population of ${ }^{10} \mathrm{~B}$. In principle, indirect population by single nucleon knockout followed by evaporation of the unlike nucleon may be possible, although we expect this indirect, two-step pathway to be very weak, owing to the large nucleon separation energies in the relevant $A=11$ systems and the very small predicted shell model strength for one nucleon removal to states above these first $A=11$ nucleon thresholds. High-precision (stable beam) observations of finalstate exclusive ${ }^{10} \mathrm{~B}$ momentum distributions would clarify such aspects of the reaction mechanism that are currently assumed.

\section{B. $s d$ shell: ${ }^{28} \mathrm{Mg}(-2 p)$ and ${ }^{26} \mathrm{Si}(-2 n)$}

Exotic nuclei with valence nucleons in the $s d$ shell have been the focus of several two-nucleon removal experiments studying the evolution of structure away from the valley of $\beta$ stability. The initial- and final-state structures are often well described within conventional $s d$-shell model space calculations offering good test cases for studies of the reaction mechanism. Details of their residue momentum distributions could offer an additional test of the shell model and the reaction mechanism in this region.

We first review the two-proton knockout from ${ }^{28} \mathrm{Mg}$ at $83.2 \mathrm{MeV} /$ nucleon on a ${ }^{9} \mathrm{Be}$ target, previously studied in Refs. [6,9,11]. To date, this is the only experimental example with measured final-state exclusive ${ }^{26} \mathrm{Ne}$ momentum distributions. Four states were populated, being the $0^{+}$ground state, the first and second $2^{+}$states, and the first $4^{+}$state. Previous work demonstrated the significant difference between the ground state and $4^{+}$residue momentum distributions, despite strong experimental (reaction target) broadening of the measured distributions.

We comment here on the effects on the 2.02 and $3.70 \mathrm{MeV}$ $2^{+}$state momentum distributions of the subtle differences in their TNAs, tabulated in Ref. [8]. To remove the small difference in the average separation energies of the protons for the two states, calculations used identical radial wave functions, but this binding effect is in practice negligible. The calculated widths of the residue momentum distributions are different by $\sim 10 \%$. Clearly a higher statistics experiment would be required to examine this difference predicted by the
TABLE III. USD shell-model TNAs for the first two $2^{+}$states in ${ }^{24} \mathrm{Si}$, populated in two-neutron knockout from ${ }^{26} \mathrm{Si}$.

\begin{tabular}{rrrrrr}
\hline \hline$J_{f}^{\pi}$ & {$\left[1 d_{5 / 2}\right]^{2}$} & {$\left[1 d_{5 / 2}, 1 d_{3 / 2}\right]$} & {$\left[1 d_{3 / 2}\right]^{2}$} & {$\left[2 s_{1 / 2}, 1 d_{3 / 2}\right]$} & {$\left[2 s_{1 / 2}, 1 d_{5 / 2}\right]$} \\
\hline $2_{1}^{+}$ & -0.70074 & 0.43499 & 0.00594 & -0.00188 & -0.02781 \\
$2_{2}^{+}$ & -0.38021 & -0.12354 & -0.12945 & -0.15876 & -0.58292 \\
\hline \hline
\end{tabular}

shell model. There are, however, other examples where the $s d$-shell model predicts TNAs that exhibit a larger degree of sensitivity, as , for example, the following.

A second specific example is the two-neutron $(T=1)$ knockout from ${ }^{26} \mathrm{Si}$. Measurements for this reaction, made at $109 \mathrm{MeV}$ per nucleon on a ${ }^{9} \mathrm{Be}$ target, were reported in Ref. [31]. Details of the nucleon radial wave functions and $S$ matrices can be found in Ref. [9]. Populations of two excited states in ${ }^{24} \mathrm{Si}$ were observed, the first $2^{+}$state at $1.86 \mathrm{MeV}$, and a state at $3.41 \mathrm{MeV}$ corresponding to a theoretically predicted $\left(2^{+}, 4^{+}\right)$doublet, with theoretical excitation energies of 3.867 and $3.962 \mathrm{MeV}$. The cross sections for these measured and theoretical states were analyzed [9] assuming that the second excited state was the second $2^{+}$state. Momentum distributions, if available, would easily distinguish between such $I=2$ and $I=4$ possibilities. Our interest here is more subtle. We consider the expected differences in the momentum distributions of the two $2^{+}$states arising from their underlying $s d$-shell model structures.

The TNAs were calculated using OXBASH within an $s d$-shell model space using the universal sd-shell (USD) interaction [32] and are presented in Table III. The TNAs calculated using the more recent USDA and USDB variants of the original USD interaction [33] were found to be very similar to the USD values. Both states have mixed $s d$-shell configurations. Inspection of the TNAs might suggest that because the second state has a stronger $\left[2 s_{1 / 2}, 1 d_{5 / 2}\right]$ configuration it may favor $L=2$ more strongly, but there is significant mixing.

Despite the strong mixing in both states, the shell-model TNAs predict each state to be populated predominately by a single and distinct total orbital angular momentum $L, L=1$ and $L=2$, respectively. The calculated $L S$-coupled twonucleon stripping partial cross sections reveal this, as is shown in Table IV. The dominance of $L=1$ and $L=2$ in these states generates the significantly different $2^{+}$state momentum distributions of Fig. 6, the $2_{2}^{+}$state having a $30 \%$ larger width. Exclusive measurements for these states would not only clarify if the second excited state is the $2_{2}^{+}$, but could also confirm the $L=2$ dominance prediction of the $s d$ shell-model calculations.

TABLE IV. Two-neutron stripping cross sections, $\sigma_{L S}$, for population of the first two $2^{+}$final states in ${ }^{24} \mathrm{Si}$. All cross sections are in $\mathrm{mb}$.

\begin{tabular}{cccccc}
\hline \hline$J_{f}^{\pi}$ & $\sigma_{11}$ & $\sigma_{20}$ & $\sigma_{21}$ & $\sigma_{31}$ & $\sigma_{\text {str }}$ \\
\hline $2_{1}^{+}$ & 0.17 & 0.02 & 0.00 & 0.00 & 0.19 \\
$2_{2}^{+}$ & 0.01 & 0.17 & 0.01 & 0.00 & 0.19 \\
\hline \hline
\end{tabular}




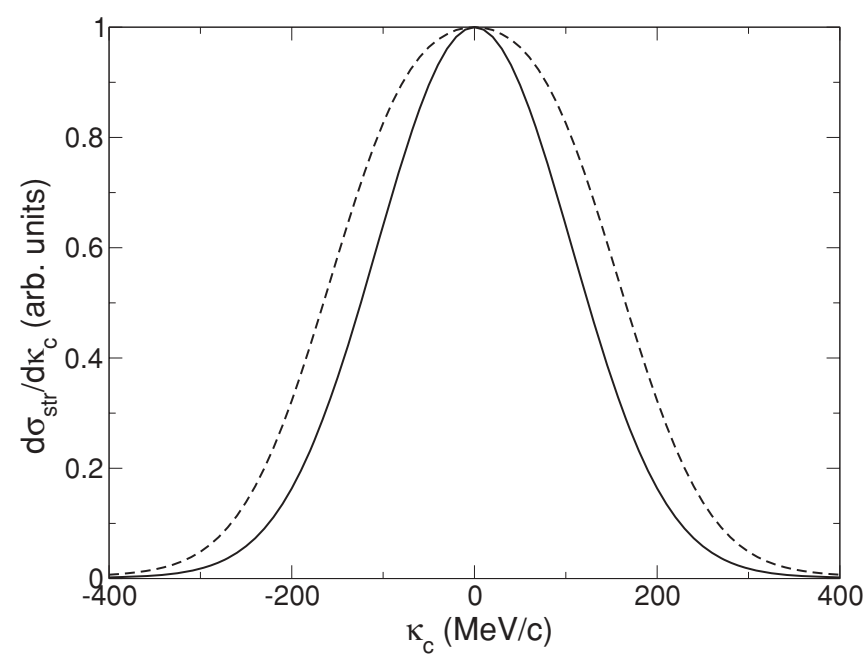

FIG. 6. Normalized residue momentum distributions for the first (solid) and second (dashed) ${ }^{24} \mathrm{Si}\left(2^{+}\right)$states populated in $2 n$ knockout from ${ }^{26} \mathrm{Si}$. Though the same $s d$-shell two-nucleon configurations contribute to each state, their TNAs result in distinct $L$ makeup and momentum distributions. The full width at half maximum peak widths are are 250 and $330 \mathrm{MeV} / c$, respectively.

To consolidate our understanding of such sensitivity, we consider a further simplified example where a single configuration is expected to dominate. We consider the two configurations $\left[1 d_{5 / 2}\right]^{2}$ and $\left[1 d_{5 / 2}, 1 d_{3 / 2}\right]$, both of which can contribute to $4^{+}$states. States with such simple configurations may not be realized in ${ }^{24} \mathrm{Si}$ because $4^{+}$states in ${ }^{24} \mathrm{Si}$ are thought to be unbound, but the example serves to illustrate the expected differences that may occur elsewhere in the $s d$ shell.

We construct the TNAs as $C_{\alpha}^{41}=\sqrt{5 / 3}$, such that $N_{f}=1$ [see Eq. (19)], and the resulting $L S$ decomposition of strengths is given in Table V. It is very clear that the $\left[1 d_{5 / 2}, 1 d_{3 / 2}\right]$ configuration weights $L=4$ significantly more strongly than does $\left[1 d_{5 / 2}\right]^{2}$ and the expectation is a wider momentum distribution. As noted in Sec. II A, in this case we would expect $L+S+T=$ even contributions to be significantly suppressed owing to the two-neutron antisymmetry and the similarity of the radial wave functions for the active spin-orbit partner orbitals. This is indeed the case, as demonstrated by the stripping cross sections of Table V. The estimated strengths, $N_{f}^{L S}$, are seen to give a reasonable guide to the expected cross sections for these single-configuration examples.

The results of the calculations, shown in Fig. 7, confirm the differences in the momentum distributions expected from our simple consideration of the $N_{f}^{L S}$. Again, the specifics of

TABLE V. $L S$-coupled strengths, $N_{f}^{L S}$, and $L S$ partial stripping cross sections for pure $\left[1 d_{5 / 2}\right]^{2}$ and pure $\left[1 d_{5 / 2}, 1 d_{3 / 2}\right]$ two-neutron configurations populating $4^{+}$final states in ${ }^{24} \mathrm{Si}$. The cross section for $L=4, S=1$ is negligible (see text).

\begin{tabular}{lcccccc}
\hline \hline$\alpha$ & $N_{f}^{31}$ & $N_{f}^{40}$ & $N_{f}^{41}$ & $\sigma_{31}$ & $\sigma_{40}$ & $\sigma_{41}$ \\
\hline$\left[1 d_{5 / 2}\right]^{2}$ & 0.8 & 0.2 & 0.0 & 0.23 & 0.09 & 0.00 \\
{$\left[1 d_{5 / 2}\right]\left[1 d_{3 / 2}\right]$} & 0.1 & 0.4 & 0.5 & 0.06 & 0.35 & 0.00 \\
\hline \hline
\end{tabular}

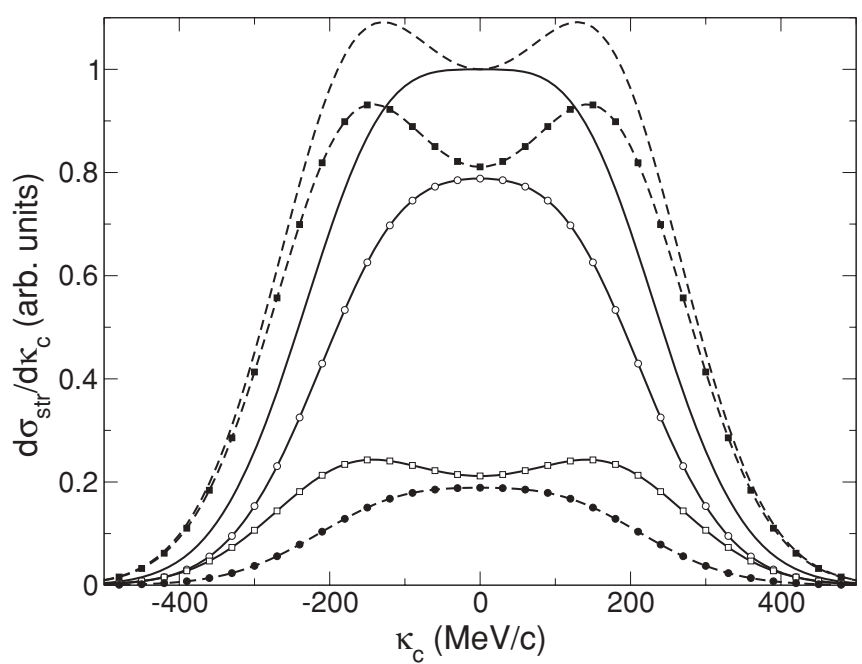

FIG. 7. Examples of the projectile rest frame residue momentum distributions for $I=4$ states in the ${ }^{26} \mathrm{Si}(-2 n)$ reaction, arising from different neutron pair configurations. The solid lines and open points assume $\left[1 d_{5 / 2}\right]^{2}$ neutron removal; the dashed lines and solid points assume $\left[1 d_{5 / 2}, 1 d_{3 / 2}\right]$ neutron removal. The full distributions are normalized to 1 at $\kappa_{c}=0$, with each contributing $L$ partial distribution scaled by the same factor. Circles show $L=3, S=1$ contributions and squares show $L=4, S=0$, with the total shown by the line. The different relative strengths of $L=3$ and $L=4$ for the two configurations generate significantly different ${ }^{24} \mathrm{Si}$ momentum distributions for states of the same $I$ (the dashed and solid curves).

the underlying structure predict considerable and observable differences in the expected residue momentum distributions.

\section{Cross shell: ${ }^{54} \mathrm{Ti}(-2 p)$}

This $(T=1)$ reaction, reported in Ref. [34], demonstrated the potential for two-nucleon knockout to probe cross-shell proton excitations in neutron-rich nuclei. In particular, a ${ }^{52} \mathrm{Ca}\left(3^{-}, 3.9 \mathrm{MeV}\right)$ state was populated in two-proton removal from ${ }^{54} \mathrm{Ti}\left(0^{+}\right)$on a ${ }^{9} \mathrm{Be}$ target at $72 \mathrm{MeV}$ per nucleon. Details of the eikonal $S$-matrices and nucleon radial wave functions can be found in Ref. [34]. Previous theoretical estimates for the $3^{-}$state yield assumed pure $\left[1 f_{7 / 2}, 2 s_{1 / 2}\right]$ or $\left[1 f_{7 / 2}, 1 d_{3 / 2}\right]$ configurations, providing an estimated upper limit for the cross section to this state as an incoherent sum of these contributions.

Taking instead a coherent sum will give (i) a different total cross section and (ii) a different residue momentum distribution. Here we assess the expected sensitivity to the relative strengths and phases of these two configurations. We calculate the two-proton stripping cross sections and momentum distributions as a function of the TNAs for $\left[1 f_{7 / 2}, 1 d_{3 / 2}\right]$ removal, $C_{f d}^{31}$, and for $\left[1 f_{7 / 2}, 2 s_{1 / 2}\right]$ removal, $C_{f s}^{31}$. For either of these pure configurations the stripping cross sections scale with $\left[C_{f \ell}^{I T}\right]^{2}$. To maintain an overall scaling when the configurations are mixed, the two amplitudes are adjusted such that

$$
\left[C_{f s}^{31}\right]^{2}+\left[C_{f d}^{31}\right]^{2}=1,
$$

with $C_{f s}^{31}$ assumed to be positive. The total incoherent strength thus remains constant. We calculate the stripping cross sections 


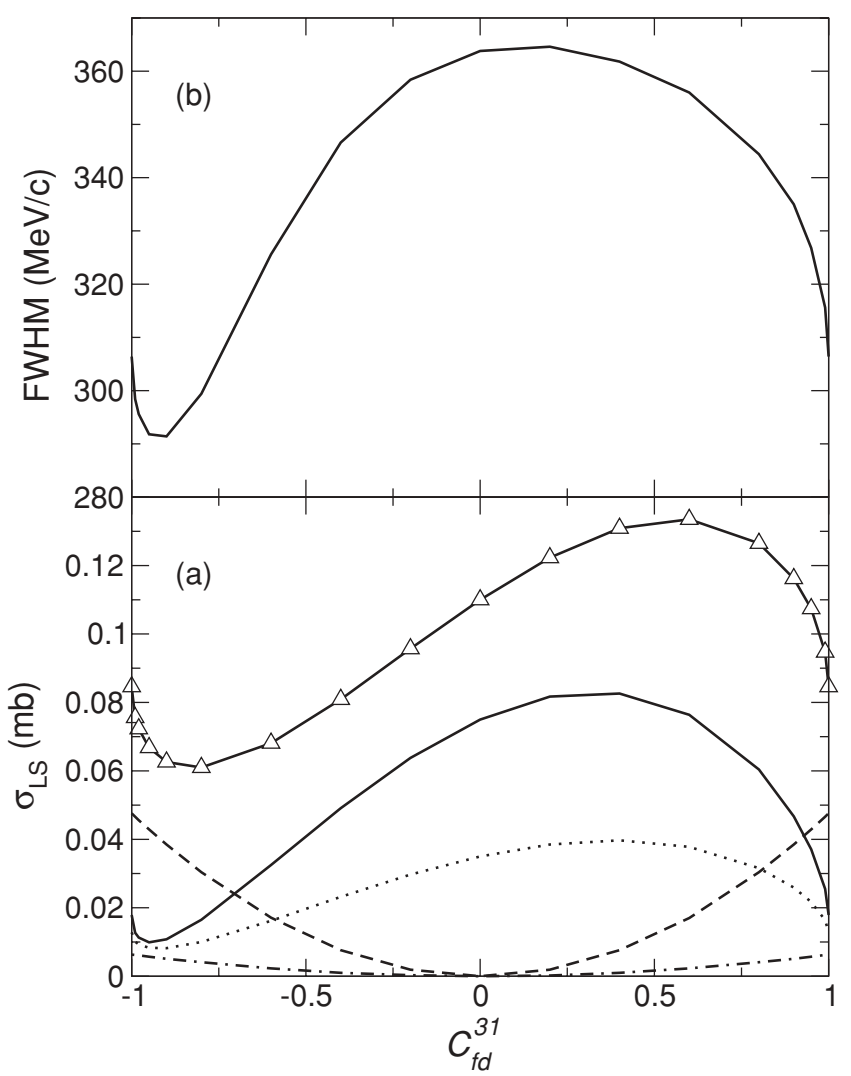

FIG. 8. (a) Two-nucleon stripping cross sections and (b) the FWHM of the residue momentum distribution when populating the ${ }^{52} \mathrm{Ca}\left(3^{-}, 3.9 \mathrm{MeV}\right)$ state. These observables are shown as a function of the amplitude $C_{f d}^{31}$. The (positive) amplitude $C_{f s}^{31}$ of the second configuration is given by Eq. (27). The bottom panel shows the partial cross sections with $\{L, S\}$ for the values $\{2,1\}$ (dashed), $\{3,0\}$ (solid), $\{3,1\}$ (dotted), and $\{4,1\}$ (dot-dashed). The total stripping cross section is shown by the solid line with open triangles. The calculations presented do not include the isospin Clebsch-Gordan coefficient $\left(C^{2}=0.846\right)$.

for each contributing $L S$ combination and the FWHM for the residue momentum distributions. This is not the whole story for the momentum distribution-there are also subtle changes of shape beyond the nominal width - but this FWHM width provides a guide to the expected behavior. The resulting calculations are shown in Fig. 8. A few points follow immediately; the $\left[1 f_{7 / 2}, 2 s_{1 / 2}\right]$ configuration only contributes to the $L=3$ cross section, giving no interference with $L=2$ and $L=4$. So these latter terms are simply proportional to $\left[C_{f d}^{31}\right]^{2}$ and are zero at the center of the plot. The $L=4$ contributions are also generally weak and the overall width of the residue momentum distribution is largely determined by the relative strengths of the $L=2$ and $L=3$ contributions.

Both the cross section and FWHM of the momentum distribution show a strong sensitivity to the mixing of the two configurations; the cross section varies by a factor of two and the width of the momentum distribution by $25 \%$. It is clear that the underlying structure and the relative strengths of the TNAs are critical to determining both the removal cross section and the shape of the momentum distribution. Of interest

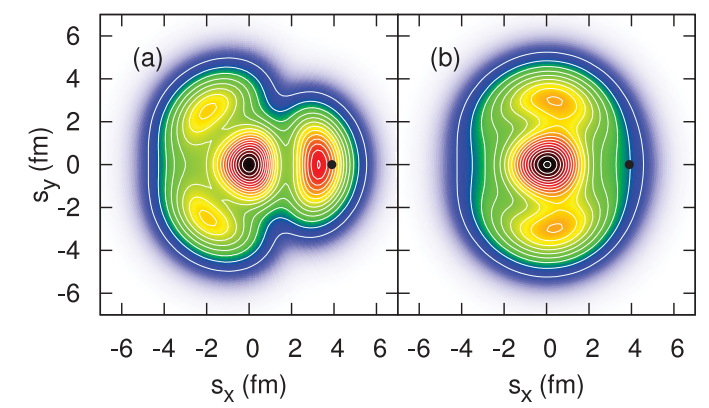

FIG. 9. (Color online) Impact parameter plane-projected twonucleon joint position probabilities, for $L=3, S=0$, for (a) $C_{f d}^{31}=$ $\sqrt{2} / 2$ and (b) $C_{f d}^{31}=-\sqrt{2} / 2$ for the ${ }^{52} \mathrm{Ca}\left(3^{-}, 3.9 \mathrm{MeV}\right)$ state populated in two-proton knockout form ${ }^{54} \mathrm{Ti}$. The plot shows the probability distribution of nucleon 2 when nucleon 1 is positioned at the back circle. The color scale (white-blue-green-yellow-red-black) is the same for both plots. The source of the differences in the calculated cross sections (see Fig. 8) for these two choices of TNAs is evident in the pair correlations manifest in these projected twoparticle joint position probabilities Note the asymmetry at $\varphi_{12}=90^{\circ}$ (i.e., $s_{x}=0$ ), induced by mixing of different parity orbitals.

are the extremes of the plot, with $\left|C_{f d}^{31}\right| \approx 1$. Here both the two-nucleon removal cross sections and momentum distribution widths are acutely sensitive to the small admixtures of the $\left[1 f_{7 / 2}, 2 s_{1 / 2}\right]$ configuration, but also strongly dependent on its sign. If the two amplitudes are of opposite phase, then both the cross section and width decrease rapidly. Conversely, they increase rapidly if in phase. This is indicative of a sensitivity to small cross-shell admixtures in many cases.

We now contrast the impact parameter plane-projected twonucleon joint probability distributions, for values of $C_{f d}^{31}=$ $\pm \sqrt{2} / 2$, in Fig. 9. The difference in this cross-shell case is now striking and it is clear that taking the amplitudes to be in phase (the +ve choice) enhances the two-nucleon spatial correlations, which then drives the significantly larger twonucleon knockout cross section and differences in the residue momentum distribution.

As is clear from Fig. 8, a precise measurement of the residue momentum distribution for this reaction would allow an estimate of the relative strengths and phases of the amplitudes of the two assumed active two-nucleon (cross-shell) configurations. The knockout cross sections themselves are also shown to depend strongly on the mixing. To date, analyses of two-nucleon knockout from exotic (asymmetric) systems have shown that the theoretical cross sections overestimate those measured experimentally by of a factor of about two, quantified as $R_{S}(2 N)=\sigma_{\exp } / \sigma_{\text {theor }} \approx 0.55$ (see, e.g., Ref. [9]). This suppression effect thus introduces an ambiguity in the absolute cross sections that is significant at the level of the differences being shown in Fig. 8. Such suppressions of the cross sections predicted using the shell-model spectroscopy may themselves be, at least in part, a manifestation of the use of TNAs calculated in a truncated shell-model space and that exclude a large number of (small-amplitude) cross-shell configurations. Based on the limited measurements available to date, there is no indication that the (missing) physics that drives the suppression of cross-section strength has implications for 
the shape of the residue momentum distribution. In addition, more accurate exclusive final-state data are needed to assess these expectations further.

\section{SUMMARY}

We discuss the momentum distributions of the heavy residues after two-nucleon knockout reactions using $L S$ coupling. The main factor determining the width of these momentum distributions is shown to be the transferred total orbital angular momenta $L$ of the two nucleons. We provide insight into the expected widths of momentum distributions from the removal of the nucleon pair from different configurations showing that information can be gained from and upon the strengths of the theoretical TNAs and the contributing $L$ they generate. The unambiguous observation of effects associated with specific pairs of nucleon orbitals may require transitions to final states that are relatively pure or simple configurations. Some illustrative examples are presented and discussed.

The conclusion of previous work - that the residue momentum distribution was simply characteristic of the final-state spin-is considered in further detail. It is true that, generally, higher-spin final states will lead to wider residue momentum distributions, but the details of the shell-model two-nucleon overlap are important in understanding the details of the residue momentum distributions. Quantitative testing and confirmation of such sensitivity to the underlying structure will be essential for the exploitation of two-nucleon knockout methods and their extension for deformed nuclei.

The critical importance of configurations of different parity in enhancing pairing correlations is demonstrated by consideration of the angular correlations inherent in the two-nucleon density. While discussed here in the context of two-nucleon removal reactions and enhancements of two-nucleon-removal cross sections, such considerations of large basis TNAs and the importance of small admixtures of different parity are entirely general. In the context of the suppression of shellmodel strength, previous studies with radioactive beams have demonstrated that the theoretical cross sections overestimate experiment by about a factor of two. It will be important to experimentally verify the influence of cross-shell excitations on structurally better-understood cases, such as for ${ }^{12} \mathrm{C},{ }^{16} \mathrm{O}$, and ${ }^{40} \mathrm{Ca}$, to clarify the extent to which the necessary reductions may depend (in part) on the truncated model spaces used. It will also be important to further assess the importance of cross-shell proton excitations in studies of islands of inversion using the two-proton knockout methodology (see, e.g., Refs. [35-37]), where very strong reductions of two-proton knockout cross sections are observed.

Here our emphasis has been on light and medium mass projectiles. Another interesting example is the two-proton removal reaction from ${ }^{208} \mathrm{~Pb}$; not only are there a large number of active orbitals, producing a plethora of states, but the majority of states are good two-proton hole configurations with minimal mixing.

The study of such reactions with odd-mass projectiles brings an added layer of complication, with, typically, each final state being populated via several nucleon-pair total angular momenta. The widths of the residue momentum distributions are then no longer simply related to a single final-state spin. However, the underlying structure sensitivity discussed here may still yield characteristic widths for different final states in the same residue, somewhat independent of the final-state spin.

\section{ACKNOWLEDGMENTS}

This work was supported by the United Kingdom Science and Technology Facilities Council (STFC) through Research Grant No. ST/F012012. ECS gratefully acknowledges support from the United Kingdom Engineering and Physical Sciences Research Council under Grant No. EP/P503892/1.
[1] P. G. Hansen, Phys. Rev. Lett. 77, 1016 (1996).

[2] F. Barranco and E. Vigezzi, in Break-up of Halo States Induced by Nuclear Interactions, edited by R. A. Broglia and P. G. Hansen (World Scientific, Singapore, 1998), p. 217.

[3] P. G. Hansen and J. A. Tostevin, Annu. Rev. Nucl. Part. Sci. 53, 219 (2003).

[4] C. A. Bertulani and P. G. Hansen, Phys. Rev. C 70, 034609 (2004).

[5] A. Gade et al., Phys. Rev. C 77, 044306 (2008).

[6] D. Bazin et al., Phys. Rev. Lett. 91, 012501 (2003).

[7] J. A. Tostevin, Eur. Phys. J. Spec. Top. 150, 67 (2007).

[8] J. A. Tostevin, G. Podolyák, B. A. Brown, and P. G. Hansen, Phys. Rev. C 70, 064602 (2004).

[9] J. A. Tostevin and B. A. Brown, Phys. Rev. C 74, 064604 (2006).

[10] E. C. Simpson and J. A. Tostevin, Phys. Rev. C 79, 024616 (2009).

[11] E. C. Simpson, J. A. Tostevin, D. Bazin, and A. Gade, Phys. Rev. C 79, 064621 (2009).

[12] E. C. Simpson, J. A. Tostevin, D. Bazin, B. A. Brown, and A. Gade, Phys. Rev. Lett. 102, 132502 (2009).

[13] P. G. Hansen and J. A. Tostevin, Annu. Rev. Nucl. Part. Sci. 53, 219 (2003).
[14] C. A. Bertulani and C. De Conti, Phys. Rev. C 81, 064603 (2010).

[15] N. C. Summers, J. S. Al Khalili, and R. C. Johnson, Phys. Rev. C 66, 014614 (2002).

[16] R. J. Glauber, in Lectures in Theoretical Physics, edited by W. E. Brittin and L. G. Dunham (Interscience Publishers, New York, 1959), Vol. 1, p. 315.

[17] J. S. Al Khalili, J. A. Tostevin, and I. J. Thompson, Phys. Rev. C 54, 1843 (1996).

[18] J. A. Tostevin, J. Phys. Conf. Ser. 49, 21 (2006).

[19] G. F. Bertsch, R. A. Broglia, and C. Riedel, Nucl. Phys. A 91, 123 (1967).

[20] W. T. Pinkston, Phys. Rev. C 29, 1123 (1984).

[21] F. A. Janouch and R. J. Liotta, Phys. Rev. C 27, 896 (1983).

[22] F. Catara, A. Insolia, E. Maglione, and A. Vitturi, Phys. Rev. C 29, 1091 (1984).

[23] F. Catara, A. Insolia, E. Maglione, and A. Vitturi, Phys. Lett. B 149, 41 (1984).

[24] A. Insolia, R. J. Liotta, and E. Maglione, J. Phys. G 15, 1249 (1989).

[25] M. A. Tischler, A. Tonina, and G. G. Dussel, Phys. Rev. C 58, 2591 (1998). 
[26] B. A. Brown, Phys. Rev. C 58, 220 (1998).

[27] B. A. Brown et al., Oxbash for Windows, MSU-NSCL Report No. 1289, 2004.

[28] E. K. Warburton and B. A. Brown, Phys. Rev. C 46, 923 (1992).

[29] B. A. Brown, P. G. Hansen, B. M. Sherrill, and J. A. Tostevin, Phys. Rev. C 65, 061601 (2002).

[30] J. A. Tostevin, P. Batham, G. Podolyák, and I. J. Thompson, Nucl. Phys. A 746, 166c (2004).
[31] K. Yoneda et al., Phys. Rev. C 74, 021303(R) (2006).

[32] B. H. Wildenthal, Prog. Part. Nucl. Phys. 11, 5 (1984).

[33] B. A. Brown and W. A. Richter, Phys. Rev. C 74, 034315 (2006).

[34] A. Gade et al., Phys. Rev. C 74, 021302 (2006).

[35] A. Gade et al., Phys. Rev. Lett. 99, 072502 (2007).

[36] P. Adrich et al., Phys. Rev. C 77, 054306 (2008).

[37] P. Fallon et al., Phys. Rev. C 81, 041302(R) (2010). 